\title{
Excess genistein suppresses the synthesis of extracellular matrix in female rat mandibular condylar cartilage
}

\author{
Shi-bin YU\#, Xiang-hui XING\#, Guang-ying DONG, Xi-li WENG, Mei-qing WANG* \\ School of Stomatology, Fourth Military Medical University, Xi'an 710032, China
}

\begin{abstract}
Aim: To investigate the effect of excess genistein on the extracellular matrix in mandibular condylar cartilage of female rats in vivo. Methods: Female SD rats were administered through oral gavage with genistein $(50 \mathrm{mg} / \mathrm{kg}$ ) or placebo daily for 6 weeks. The morphological changes of temporomandibular joints were studied with HE staining. The expression of cartilage matrix compounds (aggrecan and collagen type II), estrogen-related molecules (aromatase, estradiol, ER $\alpha$ and ER $\beta$ ) and proliferating cell nuclear antigen (PCNA) in mandibular condylar cartilage was detected using immunohistochemistry, ELISA and real-time PCR.

Results: The genistein treatment significantly reduced the thickness of the posterior and middle regions of mandibular condylar cartilage, and decreased the expression of collagen type II, aggrecan and PCNA. Compared with the control group, the estradiol content and expression levels of the key estradiol-synthesizing enzyme aromatase in the genistein-treatment group were significantly decreased. The genistein treatment significantly increased the expression of ERB, but decreased the expression of ER $\alpha$.

Conclusion: Excess genistein suppresses extracellular matrix synthesis and chondrocytes proliferation, resulting in thinner mandibular condylar cartilage. These effects may be detrimental to the ability of mandibular condylar cartilage to adapt to mechanical loads.
\end{abstract}

Keywords: genistein; osteoarthritis; mandibular condylar cartilage; estrogen; estrogen receptors

Acta Pharmacologica Sinica (2012) 33: 918-923; doi: 10.1038/aps.2012.49; published online 18 Jun 2012

\section{Introduction}

Osteoarthritis (OA) is the most common arthropathy in elderly people, and its socio-economic impact has increased as life expectancy has risen. OA is a degenerative disease characterized by the loss of collagens and proteoglycans as the main structural molecules of articular cartilage ${ }^{[1]}$. Epidemiological studies have shown that the prevalence and incidence of OA increase after menopause and that OA symptoms are more severe in postmenopausal women than in men ${ }^{[2-4]}$, which suggests a link between OA and estrogen.

Although estrogen replacement therapy (ERT) has been proven to reduce the risk of $\mathrm{OA}^{[5-7]}$, its application is hampered by an increased risk of breast cancer, myocardial infarction, and stroke ${ }^{[8,9]}$. Therefore, using phytoestrogens, which lack the specific side-effects of estrogens, may provide an alternative therapy ${ }^{[10,11]}$. Phytoestrogens are abundant in soybean products ${ }^{[12]}$; genistein, which structurally resembles

\footnotetext{
\#These authors contributed equally to this work.

* To whom correspondence should be addressed.

E-mail mqwang@fmmu.edu.cn

Received 2011-11-18 Accepted 2012-04-16
}

$17 \beta$-estradiol, is particularly abundant. It has been shown that genistein can bind to estrogen receptors (ERs) with an affinity 100 to 1000 -fold less than that of estradiol and that it competes with estradiol and displaces it from its binding sites ${ }^{[13]}$. A clinical study has suggested that consuming $88 \mathrm{mg}$ phytoestrogen per day may benefit OA patients ${ }^{[14]}$. Hooshmand et al have also reported that genistein reduced the in vitro production of lipopolysaccharide-induced cyclooxygenase (COX)-2 in chondrocytes, indicating that genistein may be an attractive and viable alternative therapy for treating or preventing $\mathrm{OA}^{[15]}$.

The temporomandibular joint (TMJ) plays an important role in craniofacial growth and function and shows a high incidence of $\mathrm{OA}^{[16]}$. In the literature, estrogen has been shown to play an important role in the development of TMJ and temporomandibular diseases ${ }^{[17-19]}$. These findings have been supported by the existence of estrogen receptors (ERs) in mandibular condylar cartilage ${ }^{[20,21]}$.

As an estrogen-targeted tissue ${ }^{[20,21]}$, cartilage is usually a solid connective tissue that covers subchondral bone tissue and plays an important role in the development of OA. To the best of our knowledge, however, few studies of the effect of genistein on cartilage have been reported, especially in vivo studies. The 
aim of the present study was to investigate the effect of excess genistein on in vivo mandibular condylar cartilage.

\section{Materials and methods}

\section{Genistein treatment in vivo}

All the animal experimental procedures were approved by the Animal Research Committee of the Fourth Military Medical University. Thirty 7-week-old female SD rats, weighing 180-190 g, were provided by the animal center of the Fourth Military Medical University. All animals were housed at $22^{\circ} \mathrm{C}$ and $30 \%-60 \%$ relative humidity with a normal daynight rhythm (a 12:12 h light-dark cycle). The animals were randomly assigned to vehicle control and genistein-treatment groups (15 in each group). They had free access to tap water and a phytoestrogen-free diet in which corn oil was replaced with soybean oil. In the literature, the bone-protective effects of genistein (3-54 $\mathrm{mg} \cdot \mathrm{kg}^{-1} \cdot \mathrm{d}^{-1}$ ) have been reported in OVX rats, mice and postmenopausal women ${ }^{[22]}$. There have been few studies focusing on the effect of genistein on normal or intact animals. Based on a series of genistein safety studies, the no observed adverse effect level (NOAEL) for genistein in rats has been estimated to be 50 or $100 \mathrm{mg} \cdot \mathrm{kg}^{-1} \cdot \mathrm{d}^{-1}[13,23]$. Consistent with our previous study ${ }^{[22]}, 50 \mathrm{mg} \cdot \mathrm{kg}^{-1} \cdot \mathrm{d}^{-1}$ genistein was chosen as excess dose in the present study. The animals were treated once daily with genistein $(50 \mathrm{mg} / \mathrm{kg}$ body weight, $99.5 \%$ pure, Winherb Medical Science Co Ltd, China) or placebo by oral gavage. The genistein was dissolved in a placebo solution $(0.9 \% \mathrm{NaCl}, 2 \%$ Tween 80 , and $0.5 \%$ methyl cellulose in water). The application volume was $5 \mathrm{~mL} / \mathrm{kg}$ body weight. Changes in body weight during the experimental period were considered when calculating the genistein dosage. All the animals were sacrificed 6 weeks after the initiation of treatment.

\section{Tissue preparation}

For the morphological and immunohistochemistry analyses 10 rats (5 from each group) received deep anesthesia from an intraperitoneal injection of pentobarbital sodium $(50 \mathrm{mg} / \mathrm{kg}$ body weight) and were perfused with $200 \mathrm{~mL}$ of normal saline and $400 \mathrm{~mL}$ of paraformaldehyde (4\% in phosphate buffer saline, $\mathrm{pH} 7.4$ ) through their ascending aortas. The TMJs and the uterus were harvested without fatty tissue. The wet weight of uterus was measured by electron precision balance. A uterine weight index was used to measure the changes in the uterus [uterine weight index $=\mathrm{WW}(\mathrm{mg}) / \mathrm{BW}(\mathrm{g})$, where $\mathrm{WW}=$ wet weight and BW=body weight $]^{[9]}$. The TMJs were post-fixed overnight at $4{ }^{\circ} \mathrm{C}$ with the same fixative and decalcified for 1 week in Kristensen's fluid (sodium formate $52.2 \mathrm{~g}$, formic acid $174.2 \mathrm{~mL}$, and $1000 \mathrm{~mL}$ distilled water). The TMJ samples were then dehydrated in graded ethanol and embedded in paraffin. Serial $5-\mu \mathrm{m}$ mid-sagittal sections were cut parallel to the lateral surface of the condyle. To ensure a reliable comparison between the vehicle control and experimental groups, mid-sagittal sections from each joint were chosen for the morphological and immunohistochemistry analyses.

The other six rats in each group were sacrificed for the realtime PCR analysis. To obtain enough total RNA, four sets of condylar cartilages from 2 randomly selected rats constituted one sample; hence, 12 sets of condylar cartilages from 6 rats in each group were randomly assigned to 3 samples.

\section{Histological analysis}

The hematoxylin and eosin (HE) stained sections were examined under a light microscope (Leica DM2500, Wetzlar, Germany). A true-color computer-assisted image analyzing system with a digital camera (Leica DFC420, Leica, Wetzlar, Germany) and software (Qwin Plus, Leica Microsystem Imaging Solutions Ltd, Cambridge, United Kingdom) were used for image capture and measurement. The thickness of the condylar cartilage was measured in each section. The condylar cartilage was divided approximately into anterior, middle and posterior regions. In every region, the thickness was measured at three quartering points. The means of the three measurements were used for the statistical analysis.

\section{Immunohistochemical staining and analysis}

Five commercially available primary antibodies were used for the immunohistochemical staining: anti-human collagen type II goat polyclonal IgG (SC7763, Santa Cruz Biol Inc, USA); anti-rat proliferating cell nuclear antigen (PCNA) mouse monoclonal IgG (MS-106, Labvision Ltd, USA); anti-human aromatase rabbit polyclonal IgG (AB69653, Abcam, UK); antihuman ERa rabbit polyclonal IgG (SC542, Santa Cruz Biol Inc, USA); and anti-human ER $\beta$ rabbit polyclonal IgG (SC8974, Santa Cruz Biol Inc, USA). The immunohistochemical staining was conducted using a three-step avidin-biotin complex method that has been previously described ${ }^{[24]}$. The sections were incubated overnight at $4{ }^{\circ} \mathrm{C}$ with anti-human collagen type II goat polyclonal $\operatorname{IgG}(3 \mu \mathrm{g} / \mathrm{mL})$, anti-rat PCNA mouse monoclonal IgG $(3 \mu \mathrm{g} / \mathrm{mL})$, anti-human aromatase rabbit polyclonal IgG $(10 \mu \mathrm{g} / \mathrm{mL})$, anti-human ERa rabbit polyclonal IgG $(4 \mu \mathrm{g} / \mathrm{mL})$, and anti-human ER $\beta$ rabbit polyclonal IgG (3 $\mu \mathrm{g} / \mathrm{mL})$.

The immunohistochemical staining for aromatase, ERa, ER $\beta$, and PCNA was analyzed by the Qwin Plus software. Briefly, the stained sections were observed under a Leica DM2500 light microscope with a $\times 10$ objective. The measurements were performed in middle and posterior regions of the condylar cartilage, where they regularly appeared among the animals. For the aromatase, ERa and ER $\beta$ analyses, the measurement area consisted of the complete mature and hypertrophic layers in which immunoreactive signals were obvious. The positively stained region and the complete selected region covering the mature and hypertrophic layers, respectively, were measured. The percentage of the entire region that stained positive was calculated. For the PCNA sections, 4 cubic regions (300 pixels $\times 300$ pixels) were selected from approximately the middle and posterior regions of the condylar cartilage. The number of PCNA-positive chondrocytes was determined. Finally, the percent of the region that stained positive and the sum of the PCNA-positive cells in all of the cartilage were used for the statistical analysis. 
RNA preparation, reverse transcription, and real-time PCR

The mandibular condylar cartilage samples were pulverized in liquid nitrogen. The total RNA was isolated from frozen tissues using a standard TRIzol ${ }^{\circledR}$ protocol (Invitrogen, Carlsbad, CA), followed by first-strand cDNA synthesis with the RevertAid $^{\mathrm{TM}}$ First Strand cDNA Synthesis Kit (Fermentas Inc, Maryland, USA). The real-time PCR was performed in an ABI 7500 Fast thermal cycler. The protocol consisted of 40 cycles of $94^{\circ} \mathrm{C}$ for $5 \mathrm{~s}, 62^{\circ} \mathrm{C}$ for $34 \mathrm{~s}$, and $72^{\circ} \mathrm{C}$ for $1 \mathrm{~min}$ each. The specific primers were designed with the primer 3 software (Whitehead Institute for Biomedical Research, Cambridge, MA, USA) based on the cDNA sequences available at the Genebank database. Table 1 shows the sequences of primers used in this study. All the PCRs were run in triplicate for each sample, and the threshold cycles (CT) were measured. A $\triangle \mathrm{CT}$ value was calculated for each sample by subtracting the CT value of the reference gene (18S) from the CT value of the gene of interest. $18 \mathrm{~S}$ has been used as internal standard in several studies. All the samples were normalized to the $\Delta \mathrm{CT}$ value of a control sample $(\Delta \Delta \mathrm{CT})$. The relative expression of the target gene was calculated using the expression $2^{-\Delta \Delta C T}$ and is reported in arbitrary units ${ }^{[25]}$.

Table 1. Primer sequences for aggrecan, collagen type II, aromatase, $E R \alpha, E R \beta$, and $18 S$.

\begin{tabular}{llll}
\hline Gene & \multicolumn{1}{c}{ Sequence } & $\begin{array}{c}\text { Fragment } \\
\text { length (bp) }\end{array}$ & $\begin{array}{c}\text { Accession } \\
\text { number }\end{array}$ \\
\hline Aggrecan & F: 5'-CCCTCACCCCAAGAATCAAGT-3' & 178 bp & NM_022190 \\
& R: 5'- TCATTGGAGCGAAGGTTCTGG-3' & & \\
Collagen II & F: 5'-AGAACTGGTGGAGCAGCAAGA-3' 124 bp & NM_012929 \\
& R: 5'-ATCTGGACGTTAGCGGTGTG-3' & & \\
Aromatase & F: 5'-TCATCAGCAAGTCCTCGAGCA-3' & 106 bp & M33986 \\
& R: 5'-CCATTCTCGTGCATGCCAAT-3' & & \\
ER $\alpha$ & F: 5'-TGCGCAAGTGTTACGAAGTGG-3' & 108 bp & NM_012689 \\
& R: 5'-TTCGGCCTTCCAAGTCATCTC-3' & & \\
ERß & F: 5'-AAAACTCACCGTCGAGCCTT-3' & 124 bp & NM_012754 \\
& R: 5'-GCTGAATACTCATGGCGGTTG-3' & & \\
18S & F: 5'-CGGCTACCACATCCAAGGAA-3' & 187 bp & M11188 \\
& R: 5'-GCTGGAATTACCGCGGCT-3' & & \\
\hline
\end{tabular}

\section{Enzyme-linked immunosorbent assay (ELISA) for determination} of estradiol

For the ELISA analysis, the remaining 8 rats (4 from each group) were sacrificed under deep anesthesia. Two mandibular condylar cartilage sections from the same rat were used a single sample, giving 4 samples from each group. The cartilage samples were pulverized in liquid nitrogen and then placed RIPA Lysis Buffer (Beyotime Institute of Biotechnology,
Haimen, China) for $2 \mathrm{~h}$ at $4{ }^{\circ} \mathrm{C}$ for extraction. The insoluble material was removed by centrifugation at $10000 \mathrm{r} / \mathrm{min}$ for 10 min. The protein in the supernatant was estimated by the BCA method according to the manufacturer's instructions (Pierce Biotechnology Inc, Rockford, IL, USA). Forty micrograms of total protein from each group was assayed by enzyme-linked immunosorbent assay. The estradiol content was quantified by a goat anti-rat ELISA kit (QRCT-301330012133EIA\UTL, Adlitteram Diagnostic Laboratories, Inc, San Diego, CA, USA) according to the manufacturer's instructions. The minimum detectable estradiol concentration using this assay was less that $1.0 \mathrm{pg} / \mathrm{mL}$.

\section{Statistical analysis}

The SPSS 13.0 software package (SPSS Inc, Chicago, IL, USA) was used to analyze and describe the data. Student's $t$ test was used to compare values between the groups. $P$-values were considered statistically significant when less than 0.05 .

\section{Results}

\section{Excess genistein increased the uterus weight index}

The uterus weight index of the genistein-treatment group $(2.2714 \pm 0.18875)$ increased significantly $(P<0.01)$ compared to the control group $(1.7884 \pm 0.11068)$.

Excess genistein suppressed the synthesis of extracellular matrix In the mandibular condylar cartilage of both the control and genistein-treatment groups, the fibrous, proliferating, mature and hypertrophic layers were regularly arranged and had good continuity within the layers (Figure $1 \mathrm{~A}$ and 1B). The thickness of the posterior and middle regions of the condylar cartilage in genistein-treated group decreased significantly $(P<0.01)$ compared to the control group, however (Figure 1C). Consistent with the decreased cartilage thickness, the
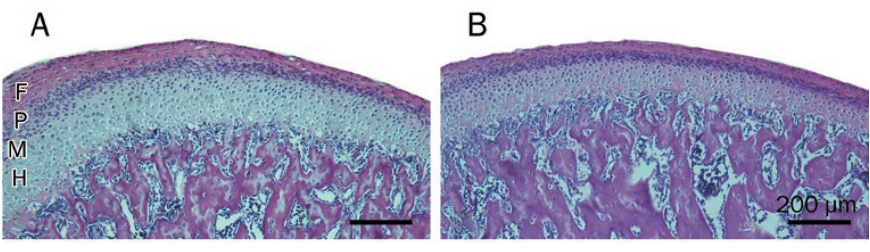

C

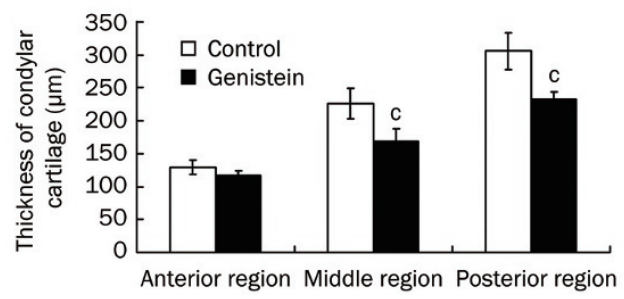

Figure 1. The histological analysis of the mandibular condylar cartilage. (A) and $(B)$ show the histological morphology in middle region of the condylar cartilage from the control and genistein treatment groups. (C) Comparison of the cartilage thickness (mean $\pm \mathrm{SD}$ ) between the genistein treatment and control groups $(n=5) .{ }^{\circ} P<0.01$ vs control. $\mathrm{F}=$ fibrous layer, $\mathrm{P}=$ proliferating layer, $\mathrm{M}=$ mature layer, $\mathrm{H}=$ hypertrophic layer. The scale bar is $200 \mu \mathrm{m}$. 
expression of collagen type II and aggrecan was lower in the genistein-treatment group than in the control group $(P<0.01)$ (Figure 2).

A

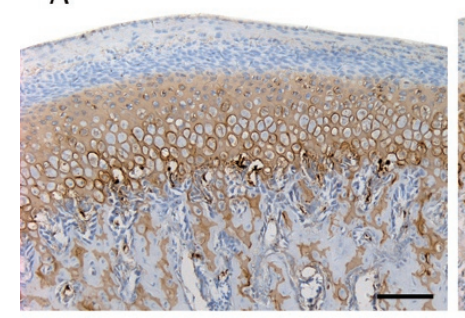

C
B

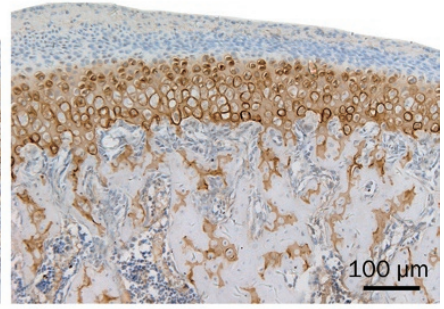

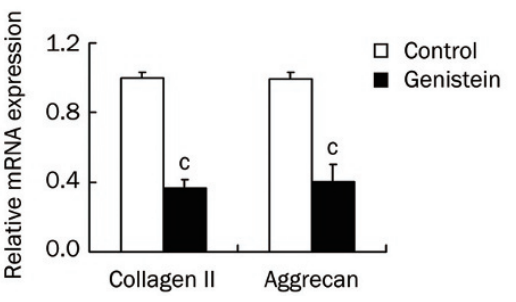

Figure 2. The expression of extracellular matrix in mandibular condylar cartilage. Panels (A) and (B) show the collagen II expression in middle region of the condylar cartilage from the control and genistein treatment groups. Panel $(\mathrm{C})$ shows the comparison of the condylar cartilage collagen II and aggrecan mRNA levels between the control and genistein treatment groups $(n=3) .{ }^{c} P<0.01$ vs control.

\section{Excess genistein suppressed the proliferation of chondrocytes}

As shown in Figure 3, the density of PCNA-positive chondrocytes was lower in the genistein-treatment group $(90.4 \pm 12.7)$ than in the control group $(217.6 \pm 28.1)(P<0.01)$.

A

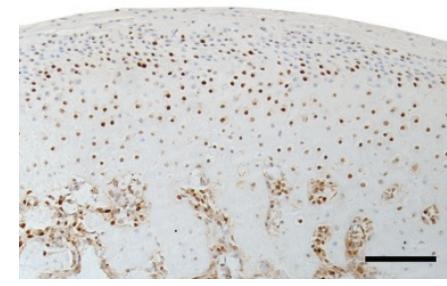

B

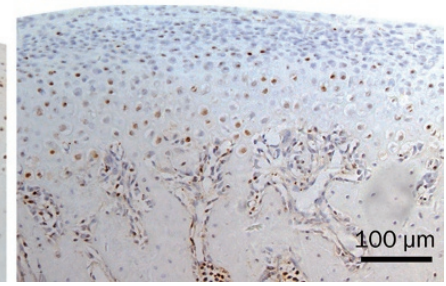

Figure 3. The PCNA expression in mandibular condylar cartilage from (A) the control group and (B) the genistein treatment group.

\section{Excess genistein decreased the level of estradiol in condylar} cartilage

The ELISA results showed that the estradiol content was lower in the genistein-treatment group $(2.1310 \pm 0.20756 \mathrm{ng})$ than in the vehicle control group $(3.4293 \pm 0.23953 \mathrm{ng})(P<0.01)$. Additionally, the expression of the key estradiol-synthesizing enzyme aromatase (as measured by both protein and mRNA levels) was lower in the genistein-treatment group $(P<0.01)$ (Figure 4) than in the control group.
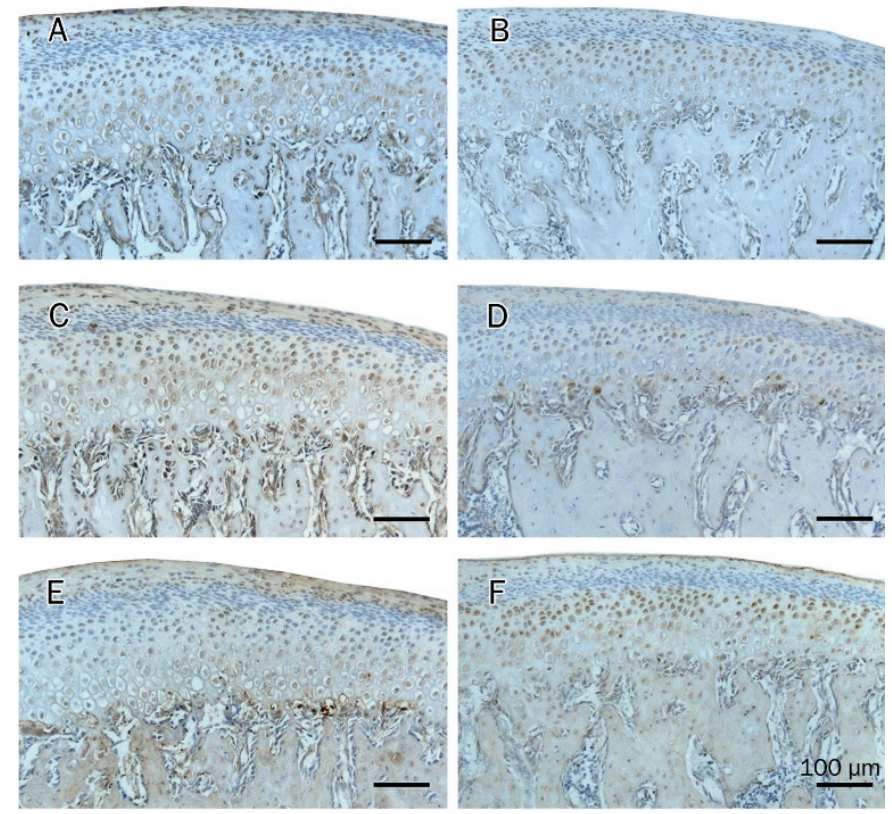

G

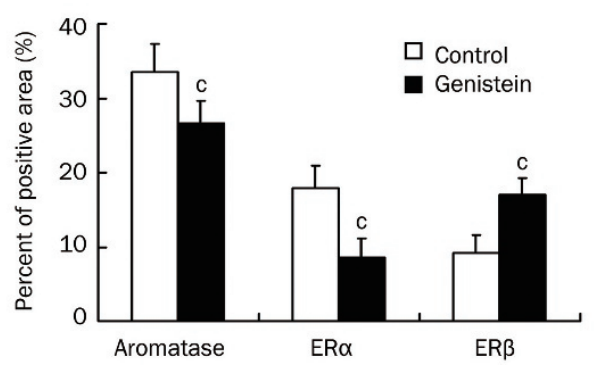

Figure 4. The expression of aromatase $(A$ and $B), E R \alpha(C$ and $D)$ and $E R B(E$ and $F$ ) in mandibular condylar cartilage. (A, C, and $E$ ) are from the control group; (B, D, and F) are from the genistein-treatment group. (G) shows the comparison of the percent of positive area between the control and genistein treatment groups $(n=5)$. ${ }^{c} P<0.01$. The scale bar is $100 \mu \mathrm{m}$.

\section{Excess genistein decreased the expression of ER $\alpha$, but increased} that of ER $\beta$

As for the ER expression, the ERa mRNA levels and immunohistochemical signals were lower in the genistein-treatment group than in the control group $(P<0.01)$. However, both the mRNA levels and immunohistochemical signals for ER $\beta$ were significantly higher in the genistein-treatment group than in the control group $(P<0.01)$ (Figure 4, 5).

\section{Discussion}

To the best of our knowledge, our study is the first to investigate the effects of genistein on mandibular condylar cartilage. Although the effect of phytoestrogens, especially genistein, on the extracellular cartilage matrix has been discussed in the literature, the data are not consistent. An in vitro study found that articular chondrocyte glycosaminoglycans (GAG) synthesis was significantly diminished following incubation with high doses of genistein $\left(10^{-5}-10^{-4} \mathrm{~mol} / \mathrm{L}\right)$ but that the level of sulfate 


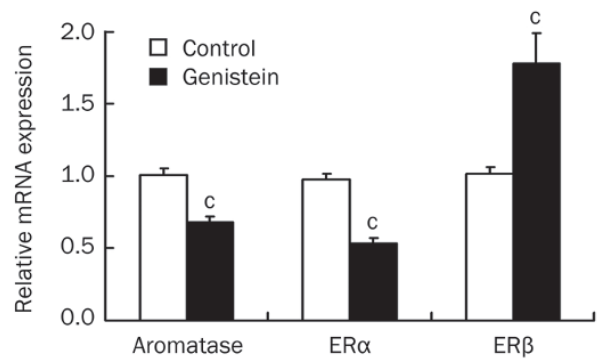

Figure 5. Comparison of the mRNA levels of aromatase, ER $\alpha$ and ER $\beta$ between the control and genistein treatment groups $(n=3) .{ }^{c} P<0.01$.

incorporation was not affected ${ }^{[1]}$. Nevertheless, Hooshmand et al found that high doses of genistein $\left(10^{-5}-10^{-4} \mathrm{~mol} / \mathrm{L}\right)$ could suppress the increased levels of YKL-40 (a marker of cartilage glycoprotein degradation) in chondrocyte supernatant that is induced by LPS treatment ${ }^{[15]}$. One preclinical study found that long-term soy phytoestrogen treatment $(129 \mathrm{mg} /$ day/person) did not have a statistically significant effect on the levels of collagen or proteoglycan in articular cartilage ${ }^{[26]}$. Our results showed that compared with the control group, both the expression of the main components of the extracellular matrix cartilage (collagen type II and aggrecan) and chondrocyte proliferation decreased significantly in genistein-treatment group, resulting in thinner mandibular condylar cartilage in the posterior and middle regions. This result is consistent with a previous report that additional estradiol $\left(10^{-8} \mathrm{~mol} / \mathrm{L}\right) \mathrm{can}$ decrease extracellular matrix synthesis, chondrocyte proliferation and the thickness of mandibular condylar cartilage in vitro ${ }^{[19]}$. Maintaining articular cartilage throughout the life of an organism plays a crucial role in establishing the biological range of cartilage adaptability ${ }^{[19]}$. Modulating cartilage thickness and extracellular matrix properties using excess genistein may create an environment more susceptible to degenerative changes.

It has been shown that chondrocytes in mandibular cartilage can locally synthesize estradiol through the activity of $\operatorname{aromatase}^{[24]}$. In the present study, both the aromatase expression and estradiol content of mandibular condylar cartilage decreased significantly after the genistein treatment, while the uterus index increased. It is possible that a particular dose of genistein can exert an estrogenic effect in cartilage by competing with local estrogen, although its effect is weak. Therefore, the need for autocrine estrogen in cartilage metabolism decreases when genistein exerts its estrogen-like activity ${ }^{[13]}$.

The literature has suggested that genistein may be completely agonistic to both ERa and ER $\beta$, although it is more potent for $\mathrm{ER} \beta$ than $\mathrm{ERa}^{[27,28]}$. It has been shown that the binding affinity of genistein was seven times greater for $\mathrm{ER} \beta$ than for $\mathrm{ERa}^{[29]}$. A recent study found that the increased ER $\beta$ expression in the genioglossus muscle after genistein treatment was much higher than that of $\mathrm{ERa}^{[9]}$. Our previous study also demonstrated that the effect of genistein on mandibular subchondral bone was predominantly mediated through $E R \beta^{[22]}$. The present study showed that ER $\beta$ expression increased significantly following treatment with excess genistein, while ERa expression decreased at both the protein and mRNA levels. These results may indicate that genistein exerts its estrogenic activity in mandibular condylar cartilage mainly through ER $\beta$, which is consistent with another recent finding that the effects of genistein on skeletal muscle major histocompatibility complex (MHC) expression was predominantly mediated through $E R \beta^{[30]}$.

In summary, the present study demonstrated that excess genistein can suppress extracellular matrix synthesis and chondrocyte proliferation in rat mandibular condylar cartilage, resulting in thinner mandibular condylar cartilage. This finding may imply decreased adaptivity to mechanical loads in mandibular condylar cartilage. Given the fluctuating differences in estrogen levels between individuals, the proper genistein dose should be studied further if genistein is to be widely used to treat OA or osteoporosis.

\section{Acknowledgements}

The authors are grateful to Ms Shu-jing CAI and Ms Cui HUA for technical assistance. This work was financially supported by the National Natural Science Foundation of China (30901699 and 30928028).

\section{Author contribution}

Mei-qing WANG and Shi-bin YU designed the study and wrote the manuscript. Shi-bin YU and Xiang-hui XING performed the animal experiments, sample preparation and immunohistochemical staining. Guang-ying DONG performed the ELISA assay and PCR and revised the manuscript. Xi-li WENG performed the HE staining and data analysis and revised the manuscript.

\section{References}

1 Claassen H, Briese V, Manapov F, Nebe B, Schünke M, Kurz B. The phytoestrogens daidzein and genistein enhance the insulin-stimulated sulfate uptake in articular chondrocytes. Cell Tissue Res 2008; 333: 71-9.

2 Bergink AP, van Meurs JB, Loughlin J, Arp PP, Fang Y, Hofman A, et al. Estrogen receptor alpha gene haplotype is associated with radiographic osteoarthritis of the knee in elderly men and women. Arthritis Rheum 2003; 48: 1913-22.

3 Spector TD, Campion GD. Generalised osteoarthritis: a hormonally mediated disease. Ann Rheum Dis 1989; 48: 523-7.

4 Ushiyama T, Ueyama H, Inoue K, Nishioka J, Ohkubo I, Hukuda S. Estrogen receptor gene polymorphism and generalized osteoarthritis. J Rheumatol 1998; 25: 134-7.

5 Felson DT, Nevitt MC. Estrogen and osteoarthritis: how do we explain conflicting study results? Prev Med 1999; 28: 445-8; discussion 449-50.

6 Nevitt MC, Cummings SR, Lane NE, Hochberg MC, Scott JC, Pressman $A R$, et al. Association of estrogen replacement therapy with the risk of osteoarthritis of the hip in elderly white women. Study of Osteoporotic Fractures Research Group. Arch Intern Med 1996; 156: 2073-80.

7 Spector TD, Nandra D, Hart DJ, Doyle DV. Is hormone replacement therapy protective for hand and knee osteoarthritis in women? The Chingford Study. Ann Rheum Dis 1997; 56: 432-4.

8 Azoulay C. Menopause in 2004: "hormone replacement therapy" is not what it used to be anymore. Rev Med Interne 2004; 25: 806-15.

9 Li W, Liu YH. Effects of phytoestrogen genistein on genioglossus 
function and oestrogen receptors expression in ovariectomized rats. Arch Oral Biol 2009; 54: 1029-34.

10 Clarkson TB, Anthony MS, Williams JK, Honore EK, Cline JM. The potential of soybean phytoestrogens for postmenopausal hormone replacement therapy. Proc Soc Exp Biol Med 1998; 217: 365-8.

11 Brzezinski A, Debi A. Phytoestrogens: the "natural" selective estrogen receptor modulators. Eur J Obstet Gynecol Rep Biol 1999; 85: 47-51.

12 Kapiotis S, Hermann M, Held I, Seelos C, Ehringer H, Gmeiner BM. Genistein, the dietary derived angiogenesis inhibitor, prevents LDL oxidation and protects endothelial cells from damage by atherogenic LDL. Arterioscler Thromb Vasc Biol 1997; 17: 2868-74.

13 McClain RM, Wolz E, Davidovich A, Edwards J, Bausch J. Reproductive safety studies with genistein in rats. Food Chem Toxicol 2007; 45: 1319-32.

14 Arjmandi BH, Khalil DA, Lucas EA, Smith BJ, Sinichi N, Hodges SB, et al. Soy protein may alleviate osteoarthritis symptoms. Phytomedicine 2004; 1: 567-75.

15 Hooshmand S, Soung do Y, Lucas EA, Madihally SV, Levenson CW, Arjmandi BH. Genistein reduces the production of proinflammatory molecules in human chondrocytes. J Nutr Biochem 2007; 18 : 609-14.

16 Okeson JP, editors. Management of temporomandibular disorders and occlusion, 5th Edition. St Lousis: Mosby Inc; 2003.

17 Yamashiro T, Takano-Yamamoto T. Differential responses of mandibular condyle and femur to oestrogen deficiency in young rats. Arch Oral Biol 1998; 43: 191-5.

18 Yasuoka T, Nakashima M, Okuda T, Tatematsu N. Effect of estrogen replacement on temporomandibular joint remodeling in ovariectomized rats. J Oral Maxillofac Surg 2000; 58: 189-96.

19 Talwar RM, Wong BS, Svoboda K, Harper RP. Effects of estrogen on chondrocyte proliferation and collagen synthesis in skeletally mature articular cartilage. J Oral Maxillofac Surg 2006; 64: 600-9.

20 Yamada K, Nozawa-Inoue K, Kawano Y, Kohno S, Amizuka N, Iwanaga $\mathrm{T}$, et al. Expression of estrogen receptor alpha (ER alpha) in the rat temporomandibular joint. Anat Rec A Discov Mol Cell Evol Biol 2003; 274: 934-41.
21 Yu SB, Wang MQ, Li YQ, Lv X, Jiang Y, Dong GY, et al. The effects of age and sex on the expression of oestrogen and its receptors in rat mandibular condylar cartilages. Arch Oral Biol 2009; 54: 479-85

22 Li YQ, Xing XH, Wang H, Weng XL, Yu SB, Dong GY. Dose-dependent effects of genistein on bone homeostasis in rats' mandibular subchondral bone. Acta Pharmacol Sin 2012; 33: 66-74.

23 Michael MR, Wolz E, Davidovich A, Pfannkuch F, Edwards JA, Bausch J. Acute, subchronic and chronic safety studies with genistein in rats. Food Chem Toxicol 2006; 44: 56-80.

24 Yu SB, Wang MQ, Zhao W, Cao HT, Liu YL. The effects of age and sex on the expression of aromatase in the rat temporomandibular joint. J Orofac Pain 2006; 20: 156-65.

25 Seibel J, Molzberger AF, Hertrampf T, Laudenbach-Leschowski U, Diel P. Oral treatment with genistein reduces the expression of molecular and biochemical markers of inflammation in a rat model of chronic TNBS-induced colitis. Eur J Nutr 2009; 48: 213-20.

26 Ham KD, Oegema TR, Loeser RF, Carlson CS. Effects of long-term estrogen replacement therapy on articular cartilage IGFBP-2, IGFBP-3, collagen and proteoglycan levels in ovariectomized cynomolgus monkeys. Osteoarthritis Cartilage 2004; 12: 160-8.

27 Casanova M, Li Y, Gaido KW, Archibeque-Engle S, Janszen DB, Heck HA. Developmental effects of dietary phytoestrogens in SpragueDawley rats and interactions of genistein and daidzein with rat estrogen receptors alpha and beta in vitro. Toxicol Sci 1999; 51: 236-44.

28 Kostelac D, Rechkemmer G, Briviba K. Phytoestrogens modulate binding response of estrogen receptors alpha and beta to the estrogen response element. J Agric Food Chem 2003; 51: 7632-5.

29 Atteritano M, Mazzaferro S, Frisina A, Cannata ML, Bitto A, D'Anna R, et al. Genistein effects on quantitative ultrasound parameters and bone mineral density in osteopenic postmenopausal women. Osteoporos Int 2009; 20: 1947-54.

30 Velders M, Solzbacher M, Schleipen B, Laudenbach U, Fritzemeier KH, Diel P. Estradiol and genistein antagonize the ovariectomy effects on skeletal muscle myosin heavy chain expression via ER-beta mediated pathways. J Steroid Biochem Mol Biol 2010; 120: 53-9. 